\title{
From many-particle interference to correlation spectroscopy
}

\author{
Mattia Walschaers, ${ }^{1,2, *}$ Jack Kuipers, ${ }^{3}$ and Andreas Buchleitner ${ }^{1, \dagger}$ \\ ${ }^{1}$ Physikalisches Institut, Albert-Ludwigs Universität Freiburg, Hermann-Herder-Strasse 3, D-79104 Freiburg, Germany \\ ${ }^{2}$ Instituut voor Theoretische Fysica, KU Leuven, Celestijnenlaan 200D, B-3001 Heverlee, Belgium \\ ${ }^{3}$ D-BSSE, ETH Zürich, Mattenstrasse 26, CH-4058 Basel, Switzerland
}

(Received 8 April 2016; published 30 August 2016)

\begin{abstract}
We show how robust statistical features of a many-particle quantum state's two-point correlations after transmission through a multimode random scatterer can be used as a sensitive probe of the injected particles' mutual indistinguishability. This generalizes Hong-Ou-Mandel interference as a diagnostic tool for many-particle transmission signals across multimode random scatterers. Furthermore, we show how, from such statistical features of the many-particle interference pattern, information can be deduced on the temporal structure of the many-particle input state, by inspection of the many-particle interference with an additional probe particle of tunable distinguishability.
\end{abstract}

DOI: 10.1103/PhysRevA.94.020104

\section{INTRODUCTION}

Identical particles are of profound importance in nature: Pauli's exclusion principle for fermions forms the cornerstone of chemistry, whereas bosonic quantum statistics allows us to prepare Bose-Einstein condensates and induces Planck's law of blackbody radiation. As such, quantum statistics describes the fundamental symmetry properties of quantum states of identical particles, in equilibrium. Yet, it turns out that the quantum dynamics of identical particles holds additional and nontrivial surprises, due to intricate interference phenomena on the level of many-particle transition amplitudes. The simplest manifestation thereof is the by now well-established Hong-Ou-Mandel (HOM) interference dip [1,2] which is observed when two photons are transmitted through a balanced beam splitter. However, it recently has been realized that HOM is only the tip of the iceberg of a whole zoo of many-particle interference phenomena [3-22], with many particles transmitted through many, randomly coupled modes as the other (truly complex) extreme, of potential relevance for photonic quantum simulation and/or computation [23-27].

Hence, many-particle interference defines a new, wide, and rather unexplored area of quantum effects which are indicative, e.g., of the entanglement properties of the many-particle input state [6,8], manifest on the semiclassical level [21], and may also be considered as novel resources, e.g., for quantum information processing [28,29]. As for all interference phenomena, however,the question remains of their robustness against decoherence effects, with partial distinguishability $[11,12,21,25,30,31]$ of the interfering particles as its arguably most prominent source. Again, HOM and many-particle generalizations thereof already provide answers for simple topologies of the coupled modes $[4,10,15,16]$.

But how does partial distinguishability impact statistical quantifiers $[9,12,32,33]$ of the fine structure of many-particle interference, in cases where the complexity of the dynamics (measured by the number of many-particle amplitudes

\footnotetext{
*mattia@itf.fys.kuleuven.be

†a.buchleitner@physik.uni-freiburg.de
}

which are dynamically superimposed) prevents a deterministic description, as in principle given, e.g., by evaluation of the full counting statistics? And to which extent can also such statistical quantifiers then be employed as diagnostic tools? Here we provide the framework for a systematic approach to these questions.

\section{MODEL}

To do so in a concrete way, we focus on a photonic setup [34]. Let us first collect the essential technical tools: Bosonic Fock space is constructed [35,36] by the vacuum state $\Omega$ acted upon by creation operators of type $a_{j}^{\dagger}(\psi)$. The latter creates a photon in the $j$ th input mode of the linear optical circuit depicted in Fig. 1, with the argument $\psi \in \mathcal{H}_{\text {add }}$ a state vector from an auxiliary Hilbert space $\mathcal{H}_{\text {add }}$, which summarizes all additional degrees of freedom of the photon, such as the temporal structure of the incoming wave packets sketched in the figure. With the adjoint annihilation operators $a_{j}(\phi)$, the associated commutation relations read [36]

$$
\left[a_{i}(\phi), a_{j}^{\dagger}(\psi)\right]=\delta_{i j}\langle\phi, \psi\rangle .
$$

The action of the optical circuit in Fig. 1 is described by an $m \times m$ unitary matrix $U$, with $m$ the number of modes,

$$
a_{j}^{\dagger}(\psi) \mapsto \sum_{k=1}^{m} U_{j k} a_{k}^{\dagger}(\psi) .
$$

Thus, $U$ mixes the different modes while leaving the additional degrees of freedom untouched. An initial state of $n$ photons, prepared in $n$ distinct input modes $q_{1}, \ldots, q_{n}$, undergoes the dynamical mapping

$$
\begin{aligned}
& a_{q_{1}}^{\dagger}\left(\psi_{1}\right) \cdots a_{q_{n}}^{\dagger}\left(\psi_{n}\right) \Omega \\
& \quad \mapsto \sum_{k_{1}, k_{2}, \ldots, k_{n}=1}^{m} U_{q_{1} k_{1}} \cdots U_{q_{n} k_{n}} a_{k_{1}}^{\dagger}\left(\psi_{1}\right) \cdots a_{k_{n}}^{\dagger}\left(\psi_{n}\right) \Omega=: \Psi
\end{aligned}
$$

where we are particularly interested in situations where particle and mode numbers $n$ and $m$, respectively, are significantly 


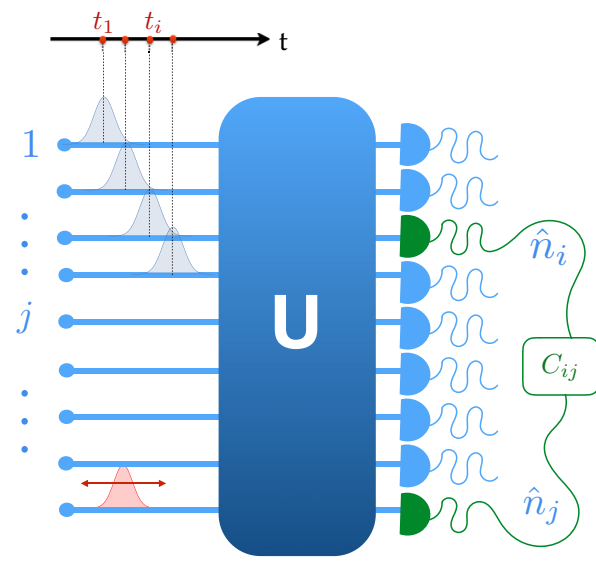

FIG. 1. Sketch of the proposed setup. $m$ input modes are connected by a linear optical circuit to $m$ output modes, on each of which a photon counter is mounted. The initial input state to be characterized consists of $n$ (here four-topmost modes on the left) photons which are described by wave packets. The photons are injected at possibly distinct times $t_{j}$, which modulates their mutual indistinguishabilities. The two-point truncated correlation function $C_{i j}$ [Eq. (4)] is the experimental observable to be sampled over all $i \neq j$. A fifth photon (bottom left) may be injected with controlled arrival time, to probe the temporal structure of the four-photon input state, through "correlation resonances," e.g., of the normalized mean $\mathrm{NM}$ of the set of $C_{i j}$ (the C-dataset) [see Eq. (13) and Fig. 4].

larger than in the HOM setting, i.e., $n, m>2$, but nevertheless far below the thermodynamic limit. Hence we explore a widely uncharted parameter regime where one may expect to uncover new physical phenomena, since it is in this parameter range that quantum granularity should be most prominent.

For $n$ and $m$ sufficiently large, and $U$ lacking any prominent symmetry properties, a deterministic evaluation of $\Psi$ rapidly turns into an intractable problem, and a statistical treatment is needed [19,32,37]. We have shown earlier that statistical sampling over the set of two-mode truncated correlation functions

$$
C_{i j}:=\left\langle\hat{n}_{i} \hat{n}_{j}\right\rangle_{\Psi}-\left\langle\hat{n}_{i}\right\rangle_{\Psi}\left\langle\hat{n}_{j}\right\rangle_{\Psi}
$$

for all pairs of output modes $i \neq j$, allows the detection of robust and characteristic features indicative of the manyparticle interferences as induced by $U$ for distinguishable and indistinguishable particles [32]. Here we expand this theory to monitor the continuous (quantum classical, in the sense of quantum statistics) transition from strictly indistinguishable to fully distinguishable particles, which can be tuned by a continuous degree of freedom accommodated by $\mathcal{H}_{\text {add }}$. Specifically, we choose this degree of freedom as given by the photon arrival times $t_{j}, j=1, \ldots, n$ (see Fig. 1).

To evaluate (4) while taking account of the temporal degree of freedom attached to each of the interfering photons, we define the single mode number operators on the output by

$$
\hat{n}_{i}:=\sum_{k} a_{i}^{\dagger}\left(\eta_{k}\right) a_{i}\left(\eta_{k}\right)
$$

where the $\eta_{k}$ form a basis of $\mathcal{H}_{\text {add }}$ [38]. The explicit expression for (4) then reads

$$
\begin{aligned}
C_{i j}= & \sum_{k \neq l=1}^{n}\left|\left\langle\psi_{k}, \psi_{l}\right\rangle\right|^{2} U_{q_{k} i} U_{q_{l} j} U_{q_{l} i}^{*} U_{q_{k} j}^{*} \\
& -\sum_{k=1}^{n} U_{q_{k} i} U_{q_{k} j} U_{q_{k} i}^{*} U_{q_{k} j}^{*},
\end{aligned}
$$

with $\psi_{k}$ the $k$ th photon's wave function in the temporal degree of freedom. The overlap $\left|\left\langle\psi_{k}, \psi_{l}\right\rangle\right|^{2}$, tantamount to the degree of indistinguishability of the $k$ th and $l$ th photon (with values between one and zero), gives a tunable weight to the two-particle interference term in (6), and thus continuously interpolates between the fully indistinguishable $\left(\left|\left\langle\psi_{k}, \psi_{l}\right\rangle\right|^{2}=1\right)$ and the fully distinguishable $\left(\left|\left\langle\psi_{k}, \psi_{l}\right\rangle\right|^{2}=0\right.$, for all $\left.k \neq l\right)$ case.

\section{STATISTICAL CERTIFICATION OF PARTIAL DISTINGUISHABILITY}

\section{A. Certification}

Given that the statistics, and, in particular, already the lowest-order moments of the $C$-dataset [32] (defined as the sample of all $C_{i j}, i \neq j$ ) define unambiguous benchmarks for many-particle interference of (in)distinguishable particles, (6) now is the fundamental building block to derive analytic expressions for those lowest-order moments, for arbitrary choices of the injected photons' mutual indistinguishabilities $\left|\left\langle\psi_{k}, \psi_{l}\right\rangle\right|^{2}$ : The normalized mean (NM) and the coefficient of variation $(\mathrm{CV})$ of the $\mathrm{C}$-dataset given by

$$
\begin{aligned}
\mathrm{NM} & :=\frac{m^{2}}{n} M_{1}, \\
\mathrm{CV} & :=\frac{\sqrt{M_{2}-M_{1}^{2}}}{M_{1}},
\end{aligned}
$$

with

$$
\begin{aligned}
& M_{1}:=\frac{2}{m(m-1)} \sum_{i<j=1}^{m} C_{i j}, \\
& M_{2}:=\frac{2}{m(m-1)} \sum_{i<j=1}^{m}\left(C_{i j}\right)^{2},
\end{aligned}
$$

together with the overlap of Gaussian photonic wave packets, centered at $t_{j}$ with spectral width $\Delta \omega$,

$$
\left|\left\langle\psi_{k}, \psi_{l}\right\rangle\right|^{2}=\exp \left(-\frac{(\Delta \omega)^{2}\left(t_{k}-t_{l}\right)^{2}}{2}\right),
$$

lead to explicit random matrix theory (RMT) [39] predictions, for $U$ given by a random unitary matrix chosen from the Haar measure. From the literature [40-44] we extract the key identity

$$
\begin{aligned}
\mathbb{E}_{U} & \left(U_{a_{1}, b_{1}} \cdots U_{a_{n}, b_{n}} U_{\alpha_{1}, \beta_{1}}^{*} \cdots U_{\alpha_{n}, \beta_{n}}^{*}\right) \\
= & \sum_{\sigma, \pi \in S_{n}} V_{m}\left(\sigma^{-1} \pi\right) \prod_{k=1}^{n} \delta\left(a_{k}-\alpha_{\sigma(k)}\right) \delta\left(b_{k}-\beta_{\pi(k)}\right),
\end{aligned}
$$


for the average over $m \times m$ unitary matrices. The functions $V_{m}\left(\sigma^{-1} \pi\right)$ in (12) can be obtained via different methods, as shown in Refs. [42,43]. The combination of (12) with (6) leads to

$$
\begin{aligned}
\mathrm{NM} \approx & \mathbb{E}_{U}\left(C_{i j}\right) \frac{m^{2}}{n}=-\frac{m}{m+1}\left[1+\frac{1}{n(m-1)}\right. \\
& \left.\times \sum_{k \neq l=1}^{n} \exp \left(-\frac{(\Delta \omega)^{2}\left(t_{k}-t_{l}\right)^{2}}{2}\right)\right]
\end{aligned}
$$

and

$$
\begin{aligned}
\overline{\mathrm{NM}} & \approx \mathbb{E}_{U}\left(\overline{C_{i j}}\right) \frac{m^{2}}{n} \\
& =-\frac{m}{m+1}\left(1+\frac{n-1}{\sqrt{1+2(\Delta \omega \delta)^{2}}(m-1)}\right) .
\end{aligned}
$$

$\mathrm{NM}$ predicts the normalized mean for well-defined injection times $t_{k}$, while $\overline{\mathrm{NM}}$ assumes independently (normal) distributed photonic arrival times $t_{k}$ with zero mean and width $\delta t$, and hence implies an additional statistical average over the arrival times. In addition, (12) allows us to evaluate CV and $\overline{\mathrm{CV}}$. We use the results from Ref. [42] in a long but straightforward computation, and obtain

$$
\begin{aligned}
\mathbb{E}_{U}\left(C_{i j}^{2}\right)= & \frac{2 A-2 B(m-5)+2 D(2+6 m-n+m n)}{(m-1) m^{2}(m+1)(m+2)(m+3)} \\
& +\frac{C\left(10+m+m^{2}\right)}{(m-1) m^{2}(m+1)(m+2)(m+3)} \\
& +\frac{(m-2)(1+3 m) n+2 n^{2}+m n^{2}+m^{2} n^{2}}{(m-1) m^{2}(m+1)(m+2)(m+3)},
\end{aligned}
$$

with

$$
\begin{aligned}
& A=\sum_{\substack{k_{1}, k_{2}, l_{1}, l_{2}=1 \\
k_{1} \neq k_{2} \neq l_{1} \neq l_{2}}}^{n}\left|\left\langle\psi_{k_{1}}, \psi_{l_{1}}\right\rangle\right|^{2}\left|\left\langle\psi_{k_{2}}, \psi_{l_{2}}\right\rangle\right|^{2}, \\
& B=\sum_{\substack{k, l_{1}, l_{2}=1 \\
k \neq l_{1} \neq l_{2}}}^{n}\left|\left\langle\psi_{k}, \psi_{l_{1}}\right\rangle\right|^{2}\left|\left\langle\psi_{k}, \psi_{l_{2}}\right\rangle\right|^{2}, \\
& C=\sum_{\substack{k, l=1 \\
k \neq l}}^{n}\left|\left\langle\psi_{k}, \psi_{l}\right\rangle\right|^{4}, \\
& D=\sum_{\substack{k, l=1 \\
k \neq l}}^{n}\left|\left\langle\psi_{k}, \psi_{l}\right\rangle\right|^{2} .
\end{aligned}
$$

Moreover, we find that

$$
\begin{aligned}
\mathbb{E}_{U}\left({\overline{C_{i j}}}^{2}\right)= & \frac{2 A^{\prime}-2 B^{\prime}(m-5)+2 D^{\prime}(2+6 m-n+m n)}{(m-1) m^{2}(m+1)(m+2)(m+3)} \\
& +\frac{C^{\prime}\left(10+m+m^{2}\right)}{(m-1) m^{2}(m+1)(m+2)(m+3)} \\
& +\frac{(m-2)(1+3 m) n+2 n^{2}+m n^{2}+m^{2} n^{2}}{(m-1) m^{2}(m+1)(m+2)(m+3)},
\end{aligned}
$$

with

$$
\begin{aligned}
A^{\prime} & =\frac{n(n-1)(n-2)(n-3)}{\left[1+2(\Delta \omega \delta t)^{2}\right]}, \\
B^{\prime} & =\frac{n(n-1)(n-2)}{\left[1+2(\Delta \omega \delta t)^{2}\right]}, \\
C^{\prime} & =\frac{n(n-1)}{\left[1+2(\Delta \omega \delta t)^{2}\right]}, \\
D^{\prime} & =\frac{n(n-1)}{\sqrt{1+2(\Delta \omega \delta t)^{2}}} .
\end{aligned}
$$

We can combine these outcomes with the results for NM (12) and $\overline{\mathrm{NM}}$ (13) to determine

$$
\begin{aligned}
& \mathrm{CV}=\frac{\sqrt{\mathbb{E}_{U}\left(C_{i j}^{2}\right)-\mathbb{E}_{U}\left(C_{i j}\right)^{2}}}{\mathbb{E}_{U}\left(C_{i j}\right)}, \\
& \overline{\mathrm{CV}}=\frac{\sqrt{\mathbb{E}_{U}\left({\overline{C_{i j}}}^{2}\right)-\mathbb{E}_{U}\left(\overline{C_{i j}}\right)^{2}}}{\mathbb{E}_{U}\left(\overline{C_{i j}}\right)} .
\end{aligned}
$$

The latter leads to the RMT prediction for the second panel in Fig. 2.

\section{B. Results for fluctuating arrival times}

Let us first compare the RMT prediction (14) for $\overline{\mathrm{NM}}$ and $\overline{\mathrm{CV}}$ to numerically generated results which are obtained by direct evaluation of (9) and (10), i.e., as the averages over all possible choices of output modes of a fixed random circuit $U$, and over Gaussian distributed $t_{k}, k=1, \ldots, n$, with variable $\delta t$ and fixed $\Delta \omega$. Figure 2 shows the statistical analog of the HOM dip, as exhibited by both $\overline{\mathrm{NM}}$ and $\overline{\mathrm{CV}}$. Our analytical RMT prediction and numerical simulation agree very well. Note that residual fluctuations of the numerical result around the RMT prediction, more prominent for the coefficient of variation, will be progressively suppressed in the thermodynamic limit.

\section{Scaling behavior}

The visibility of the dip is given by the difference between the results for the distinguishable and indistinguishable case,

$$
\begin{aligned}
V_{\mathrm{NM}} & =\left|\frac{\mathrm{NM}_{\delta t \rightarrow \infty}-\mathrm{NM}_{\delta t \rightarrow 0}}{\mathrm{NM}_{\delta t \rightarrow \infty}+\mathrm{NM}_{\delta t \rightarrow 0}}\right|, \\
V_{\mathrm{CV}} & =\left|\frac{\mathrm{CV}_{\delta t \rightarrow \infty}-\mathrm{CV}_{\delta t \rightarrow 0}}{\mathrm{CV}_{\delta t \rightarrow \infty}+\mathrm{CV}_{\delta t \rightarrow 0}}\right| .
\end{aligned}
$$

The scaling behavior in $n$ and $m$ is then obtained from (14) and (20)-(26). In Fig. 3 we show how these visibilities change as a function of the number $n$ of particles, both in the regime where $m \sim n$, and where $m \sim n^{2}$ [45]. It becomes clear from the saturation or decrease of $V_{\mathrm{NM}}$, and from the monotonous increase of $V_{\mathrm{CV}}$ with $n$, that, although the statistical spread in Fig. 2 is larger for $\overline{\mathrm{CV}}$ than for $\overline{\mathrm{NM}}$, the visibility of the distinguishability transition in the former quantity scales more favorably with the system size. This implies that, specifically in the regime of larger $n$ and $m$-and hence where the RMT prediction is more accurate [32] - the clearest transition from indistinguishable to distinguishable photons is seen in $\overline{\mathrm{CV}}$. 

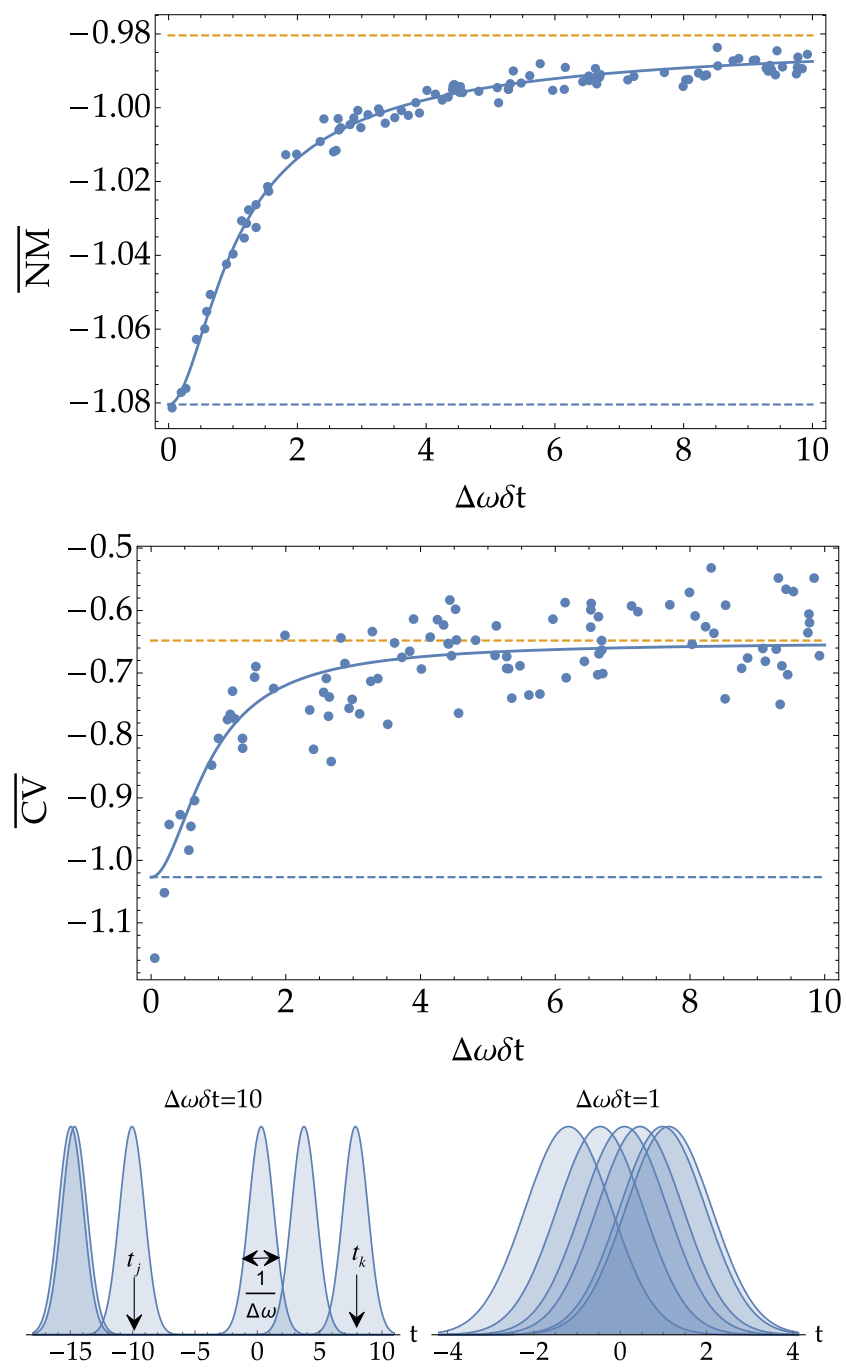

FIG. 2. Normalized mean [NM, top, Eq. (7)] and coefficient of variation $[\mathrm{CV}$, middle, Eq. (8)] of the $\mathrm{C}$-dataset generated by the transmission of $n=6$ photons across a $m=50$ mode random unitary, as a function of the temporal scatter $\delta t$ of the photons' arrival times (normally distributed, with zero mean) $t_{j}$, at given spectral width $\Delta \omega$. The bottom plot sketches two typical scenarios of the photons' timing-one where the different wave packets are typically well resolved (left) and one with a high degree of indistinguishability. Continuous lines indicate the RMT predictions (14) and (26) for $\overline{\mathrm{NV}}$ and $\overline{\mathrm{CV}}$, while dots are derived from a numerically generated C-dataset, with one single, fixed random realization of $U$, and upon average over 100 normally distributed arrival times per $t_{j}$. The differences between the predictions for strictly indistinguishable bosons and for distinguishable particles (horizontal dotted lines) determine the visibilities [(27) and (28)] of the signals.

Therefore, much as in the HOM setting, but now for large $n$ and $m$, for unknown, random $U$, and on the level of the lowest-order statistical moments of the set of two-point correlation functions read off from the n-particle output state, do these results define diagnostic tools for the experimental certification of the indistinguishable preparation of the injected photons.
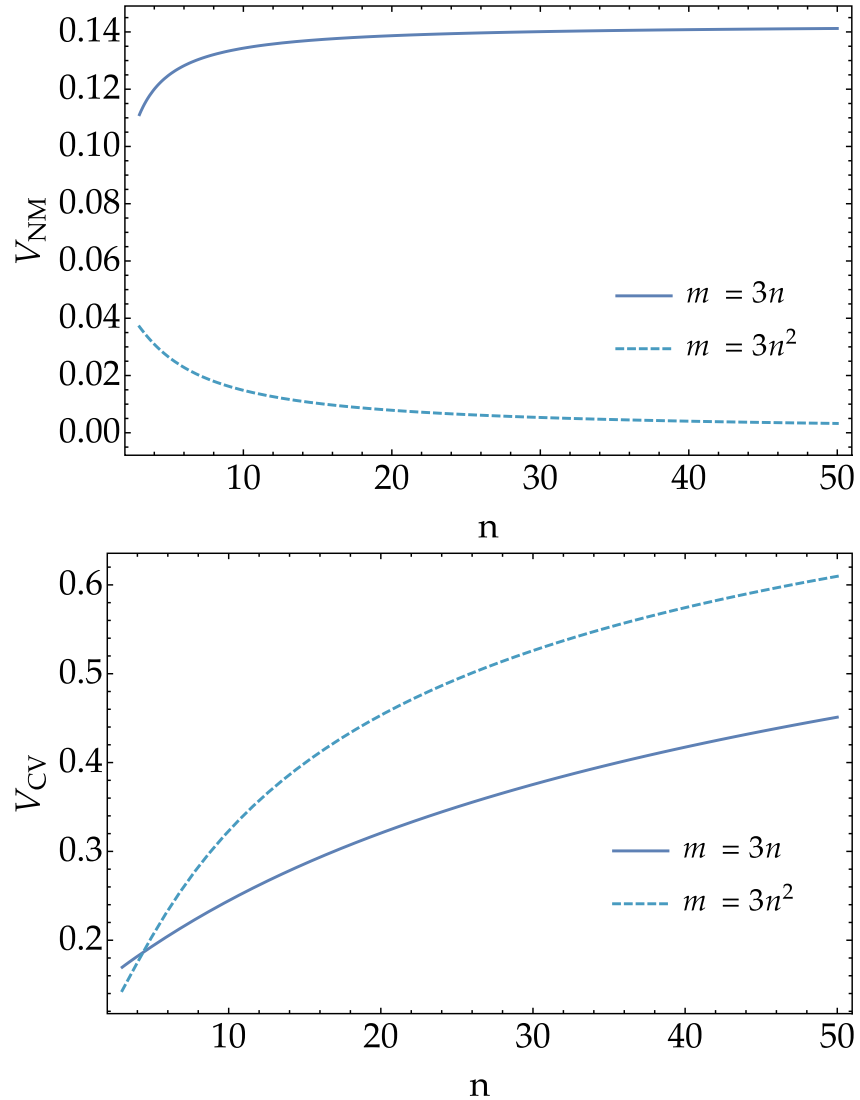

FIG. 3. RMT predictions for the visibility of normalized mean $\left[V_{\mathrm{NM}}\right.$, top, Eq. (27)] and coefficient of variation $\left[V_{\mathrm{CV}}\right.$, bottom, Eq. (28)] as obtained from (14) and (20)-(26). The number $m$ of modes is chosen to scale with the number $n$ of particles as $m=3 n$ (solid line) and as $m=3 n^{2}$ (dashed line), respectively, where the factor 3 is chosen arbitrarily.

\section{CORRELATION SPECTROSCOPY}

Next, we exploit the structure of (13) to establish how the C-dataset can be used to probe the temporal structure of the many-particle input state by manipulating a single photon's input state: Assume that the injection times of the first $n-1$ photons be fixed, and that the $n$th photon's injection time be controllable by an adjustable delay line. Then, by virtue of the sum of exponentials in (13) [and, likewise, in the corresponding expressions (15)-(19) and (25) for $\mathrm{CV}]$, whenever $t_{n} \simeq t_{k}, n \neq k$, the thus triggered two-photon interference between photons $n$ and $k$ will induce a dip in $\mathrm{NM}$, of width $(\Delta \omega)^{-1}$, centered around $t_{k}$, much as in a typical spectroscopic experiment. This protocol thus even allows the inference (with finite resolution controlled by the photons' spectral bandwidth) of the actual timing of the injected photons.

Again, as shown in Fig. 4, RMT results and numerical simulations agree qualitatively very well, with some quantitative deviations in the vicinity of the minima of NM. We attribute these to the difference between the RMT average (13) and the contribution of the "correlation resonance" between the probe and the $k$ th input photon to the signal as generated by a specific realization of $U$. 


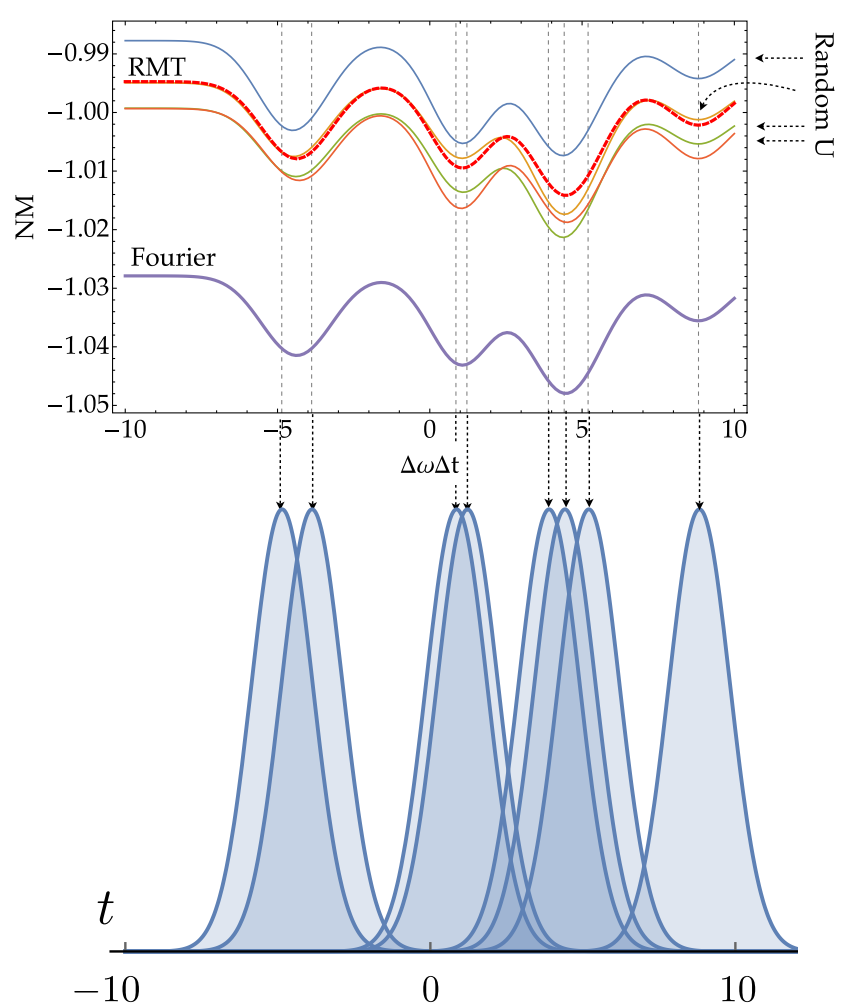

FIG. 4. Normalized mean NM, as a function of the probe photon's (see Fig. 1) delay with respect to $t=0$, and for $n-1=8$ injected photons with injection times $t_{k}=-4.86071,-3.87957,0.858186$, $1.21835,3.89386,4.41308,5.19717$, and 8.82249 (in units of $1 / \Delta \omega$ ), for numerically generated, random (thin lines) $m=30$ mode unitaries, compared to the RMT prediction (13) (thick, dashed red line), and to the result (33) for the Fourier circuit. Clearly, whenever the probe photon's delay coincides with any one of the other photons' injection times (associated wave packets are displayed in the bottom panel), a resonancelike dip emerges in the C-dataset's lowest-order statistical moment.

We finally stress that the statistical characterization of the quantum classical transition as proposed here can also be applied to structured or highly symmetric circuits such as described by Fourier matrices. These types of circuits exhibit prominent interference effects which have been experimentally demonstrated very recently [46] and can be understood analytically $[7,13,47]$. Also the $C_{i j}$ and, subsequently, NM can be directly evaluated, without recourse to RMT. NM of the Fourier circuit C-dataset is obtained via a direct evaluation of (9), with $U$ a Fourier matrix, hence

$$
U_{q_{k} i}=\frac{1}{\sqrt{m}} \exp \left(2 \pi \mathrm{i} \frac{\left(q_{k}-1\right)(i-1)}{m}\right) .
$$

We may now write (6) as

$$
\begin{aligned}
C_{i j}= & -\frac{n}{m^{2}}-\frac{1}{m^{2}} \sum_{k \neq l=1}^{n}\left|\left\langle\psi_{k}, \psi_{l}\right\rangle\right|^{2} \\
& \times \exp \left(2 \pi \mathrm{i} \frac{\left(q_{l}-q_{k}\right)(j-i)}{m}\right),
\end{aligned}
$$

which needs to be averaged over all output modes $i$ and $j$ to obtain $M_{1}(9)$. When we consider a fixed value $i$, we obtain that

$$
\sum_{\substack{j=1 \\ j \neq i}}^{m} \exp \left(2 \pi \mathrm{i} \frac{\left(q_{l}-q_{k}\right) j}{m}\right)=-\exp \left(2 \pi \mathrm{i} \frac{\left(q_{l}-q_{k}\right) i}{m}\right) .
$$

The identity (31) implies

$$
\sum_{\substack{i, j=1 \\ i \neq j}}^{m} \exp \left(2 \pi \mathrm{i} \frac{\left(q_{l}-q_{k}\right)(j-i)}{m}\right)=-m
$$

and, hence, with (7) and (9), one obtains

$$
\mathrm{NM}=-1-\frac{1}{n(m-1)} \sum_{k \neq l=1}^{n} \exp \left(-\frac{\Delta \omega^{2}\left(t_{k}-t_{l}\right)^{2}}{2}\right)
$$

with a slightly increased visibility of the signal displayed in Fig. 4, as compared to the result for a random scatterer.

Note that this result nicely illustrates two rather complementary aspects of multiparticle, multimode interference in the presence of symmetries: On the one hand, the Fourier circuit's symmetries induce the suppression of specific, well-defined output events, which define a highly sensitive probe of the precise implementation of the Fourier map and of the concomitant multiparticle interference. On the other hand, irrespective of these isolated output events specific to the Fourier map, there are robust statistical features proper to all output events which both highly symmetric and fully random circuits have in common. However, even on the level of these statistical quantifiers does the difference between the underlying unitaries emerge, through an essentially constant shift, as evident from Fig. 4. The precise connection between the specific structure of the unitaries and the observed bias remains to be elucidated.

\section{CONCLUSIONS}

Let us conclude with the observation that the treatment of many-particle interference phenomena in terms of a set of correlators with statistical properties which are indicative of structural properties of the injected quantum states defines a type of correlation spectroscopy. While we focused here on the photonic context which originally motivated this work, the underlying theoretical structure as incarnated by (6) is rather general and lends itself to straightforward generalizations to other "distinguishing" degrees of freedom, as well as to other, e.g., fermionic particle species. Since the overlaps $\left|\left\langle\psi_{k}, \psi_{l}\right\rangle\right|^{2}$ in the distinguishing degree of freedom define some sort of which-way information on the level of two-particle transition amplitudes, this furthermore indicates different directions for the decoherence theory of quantum systems of indistinguishable particles. 


\section{ACKNOWLEDGMENTS}

The authors thank Juan Diego Urbina and Klaus Richter for fruitful discussions. M.W. expresses gratitude to the
German National Academic Foundation for financial support. A.B. acknowledges support by the EU Collaborative project QuProCS (Grant Agreement No. 641277).
[1] C. K. Hong, Z. Y. Ou, and L. Mandel, Phys. Rev. Lett. 59, 2044 (1987).

[2] Y. H. Shih and C. O. Alley, Phys. Rev. Lett. 61, 2921 (1988).

[3] R. A. Campos, B. E. A. Saleh, and M. C. Teich, Phys. Rev. A 40, 1371 (1989).

[4] A. V. Belinskii and D. N. Klyshko, Sov. Phys. JETP 75, 606 (1992).

[5] Y. L. Lim and A. Beige, New J. Phys. 7, 155 (2005).

[6] C. W. J. Beenakker, J. W. F. Venderbos, and M. P. van Exter, Phys. Rev. Lett. 102, 193601 (2009).

[7] M. C. Tichy, M. Tiersch, F. de Melo, F. Mintert, and A. Buchleitner, Phys. Rev. Lett. 104, 220405 (2010).

[8] N. Cherroret and A. Buchleitner, Phys. Rev. A 83, 033827 (2011).

[9] K. Mayer, M. C. Tichy, F. Mintert, T. Konrad, and A. Buchleitner, Phys. Rev. A 83, 062307 (2011).

[10] M. C. Tichy, H.-T. Lim, Y.-S. Ra, F. Mintert, Y.-H. Kim, and A. Buchleitner, Phys. Rev. A 83, 062111 (2011).

[11] M. C. Tichy, Ph.D. thesis, Albert-Ludwigs Universität Freiburg, 2011.

[12] K. Mayer, Diploma thesis, Albert-Ludwigs Universität Freiburg, 2012.

[13] M. C. Tichy, M. Tiersch, F. Mintert, and A. Buchleitner, New J. Phys. 14, 093015 (2012).

[14] P. B. R. Nisbet-Jones, J. Dilley, A. Holleczek, O. Barter, and A. Kuhn, New J. Phys. 15, 053007 (2013).

[15] Y.-S. Ra, M. C. Tichy, H.-T. Lim, O. Kwon, F. Mintert, A. Buchleitner, and Y.-H. Kim, Proc. Natl. Acad. Sci. USA 110, 1227 (2013).

[16] Y.-S. Ra, M. C. Tichy, H.-T. Lim, O. Kwon, F. Mintert, A. Buchleitner, and Y.-H. Kim, Nat. Commun. 4, 2451 (2013).

[17] S. Laibacher and V. Tamma, Phys. Rev. Lett. 115, 243605 (2015).

[18] V. Tamma and S. Laibacher, Phys. Rev. Lett. 114, 243601 (2015).

[19] M. Walschaers, F. Schlawin, T. Wellens, and A. Buchleitner, Annu. Rev. Condens. Matter Phys. 7, 223 (2016).

[20] D. Sokolovski, J. Siewert, and L. M. Baskin, Phys. Rev. A 93, 012705 (2016).

[21] J.-D. Urbina, J. Kuipers, S. Matsumoto, Q. Hummel, and K. Richter, Phys. Rev. Lett. 116, 100401 (2016).

[22] V. S. Shchesnovich, Phys. Rev. Lett. 116, 123601 (2016).

[23] M. Bentivegna, N. Spagnolo, C. Vitelli, F. Flamini, N. Viggianiello, L. Latmiral, P. Mataloni, D. J. Brod, E. F. Galvao, A. Crespi et al., Sci. Adv. 1, e1400255 (2015).

[24] M. A. Broome, A. Fedrizzi, S. Rahimi-Keshari, J. Dove, S. Aaronson, T. C. Ralph, and A. G. White, Science 339, 794 (2013).
[25] N. Spagnolo, C. Vitelli, M. Bentivegna, D. J. Brod, A. Crespi, F. Flamini, S. Giacomini, G. Milani, R. Ramponi, P. Mataloni et al., Nat. Photon. 8, 615 (2014).

[26] J. B. Spring, B. J. Metcalf, P. C. Humphreys, W. S. Kolthammer, X.-M. Jin, M. Barbieri, A. Datta, N. Thomas-Peter, N. K. Langford, D. Kundys et al., Science 339, 798 (2013).

[27] M. Tillmann, B. Dakić, R. Heilmann, S. Nolte, A. Szameit, and P. Walther, Nat. Photon. 7, 540 (2013).

[28] M. J. Bremner, R. Jozsa, and D. J. Shepherd, Proc. R. Soc. London, Ser. A, 467, 459 (2010).

[29] S. Aaronson and A. Arkhipov, Theory Comput. 9, 143 (2013).

[30] V. S. Shchesnovich, Phys. Rev. A 91, 013844 (2015).

[31] M. C. Tichy, Phys. Rev. A 91, 022316 (2015).

[32] M. Walschaers, J. Kuipers, J.-D. Urbina, K. Mayer, M. C. Tichy, K. Richter, and A. Buchleitner, New J. Phys. 18, 032001 (2016).

[33] L. Rigovacca, C. Di Franco, B. J. Metcalf, I. A. Walmsley, and M. S. Kim, arXiv:1604.07588.

[34] This model can easily be adapted to describe free fermions [37] by considering anticommutation relations for the creation and annihilation operators. To translate our techniques to realistic cold-atom scenarios, technical extensions in the description of the scattering compound are required.

[35] O. Bratteli and D. W. Robinson, Operator Algebras and Quantum Statistical Mechanics (Springer, Berlin, 1997).

[36] R. Alicki, in Quantum Information, Computation and Cryptography, edited by F. Benatti, M. Fannes, R. Floreanini, and D. Petritis, Lecture Notes in Physics Vol. 808 (Springer, Berlin, 2010), pp. 151-174.

[37] M. Walschaers, Ph.D. Thesis, Albert-Ludwigs Universität Freiburg and KU Leuven, 2016.

[38] Note that this definition of the number operators implies that the detectors in Fig. 1 do integrate over the photon arrival times.

[39] M. L. Mehta, Random Matrices (Elsevier/Academic, Amsterdam, 2004).

[40] S. Samuel, J. Math. Phys. 21, 2695 (1980).

[41] P. A. Mello, J. Phys. A 23, 4061 (1990).

[42] P. W. Brouwer and C. W. J. Beenakker, J. Math. Phys. 37, 4904 (1996).

[43] B. Collins and P. Śniady, Commun. Math. Phys. 264, 773 (2006).

[44] G. Berkolaiko and J. Kuipers, J. Math. Phys. 54, 112103 (2013).

[45] Note that boson sampling setups are only proven to be computationally hard for $m>n^{2}$ [29]. Thus the scaling $m \sim n^{2}$ is specifically relevant within this context.

[46] A. Crespi, R. Osellame, R. Ramponi, M. Bentivegna, F. Flamini, N. Spagnolo, N. Viggianiello, L. Innocenti, P. Mataloni, and F. Sciarrino, Nat. Commun. 7, 10469 (2016).

[47] M. C. Tichy, K. Mayer, A. Buchleitner, and K. Mølmer, Phys. Rev. Lett. 113, 020502 (2014). 